\title{
Coefficients of Functional Compositions Often Grow Smoothly
}

\author{
Edward A. Bender \\ Department of Mathematics \\ University of California, San Diego \\ La Jolla, CA 92093-0112 \\ ebender@ucsd.edu \\ E. Rodney Canfield* \\ Department of Computer Science \\ University of Georgia \\ Athens, GA 30602 \\ erc@cs.uga.edu \\ L. Bruce Richmond ${ }^{\dagger}$ \\ Department of Combinatorics and Optimization \\ University of Waterloo \\ Waterloo, Ontario CANADA N2L 3G1 \\ lbrichmond@uwaterloo.ca
}

Submitted: Oct 9, 2007; Accepted: Jan 23, 2008; Published: Feb 4, 2008

AMS Subject Classification: 05A16, 05C30

\begin{abstract}
The coefficients of a power series $A(x)$ are smooth if $a_{n-1} / a_{n}$ approaches a limit. If $A(x)=F(G(x))$ and $f_{n}^{1 / n}$ approaches a limit, then the coefficients of $A(x)$ are often smooth. We use this to show that the coefficients of the exponential generating function for graphs embeddable on a given surface are smooth, settling a problem of McDiarmid.
\end{abstract}

\footnotetext{
${ }^{*}$ Research supported by the NSA Mathematical Sciences Program.

${ }^{\dagger}$ Research support by the NSERC.
} 


\section{Introduction}

Let $A(x)=\sum a_{n} x^{n}$ have nonnegative coefficients and finite nonzero radius of convergence $\rho(A)$. Recall that $\lim \sup a_{n}^{1 / n}=1 / \rho(A)$. If $a_{n-1} / a_{n}$ approaches a limit, the limit is $\rho(A)$ and we say that the coefficients are smooth or have smooth growth. In the case of a generating function, we say the corresponding class of objects is smooth. Suppose $b_{n}$ is the number of objects of size $n$. For labeled objects we deal with an exponential generating function $B(x)=\sum b_{n} x^{n} / n$ ! the ratio of coefficients is therefore $n b_{n-1} / b_{n}$. Thus we consider the ratio $n b_{n-1} / b_{n}$ rather than $b_{n-1} / b_{n}$.

We prove the following theorem on smooth growth and present some applications. The main one is a proof that the number of graphs on a surface grows smoothly, settling a problem of McDiarmid [2].

Theorem 1 Suppose that

(a) $F(x)=\sum_{n \geq 0} f_{n} x^{n}$ and $G(x)=\sum_{n \geq 1} g_{n} x^{n}$ have nonnegative coefficients and radii of convergence $0<\rho(F)<\infty$ and $0<\rho(G)$;

(b) $\operatorname{gcd}\left\{i-j \mid g_{i} g_{j} \neq 0\right\}=1$;

(c) $G(s)=\rho(F)$ for some $0<s<\rho(G)$;

(d) $\lim _{n \rightarrow \infty} f_{n}^{1 / n}=1 / \rho(F)$, where the limit is through a sequence $S$ such that $S \cap[n, n+d]$ is nonempty for some $d=d(n)=o(n)$ and all sufficiently large $n$.

Then the coefficients of $A(x)=F(G(x))$ are smooth and, in fact, $a_{n-1} / a_{n} \sim s$ where $s$ is given by (c).

We note that smoothness of the $a_{n}$ implies $\lim a_{n}^{1 / n}=1 / \rho(A)$. To see this, observe that smoothness implies that for all $\delta>0$ there is a $K$ such that $\left|a_{k} / a_{k-1}-1 / \rho(A)\right|<\delta$ whenever $k>K$ and so

$$
a_{n}^{1 / n}=a_{K}^{1 / n} \prod_{k=K+1}^{n}\left(a_{k} / a_{k-1}\right)^{1 / n} \geq a_{K}^{1 / n}(1 / \rho(A)-\delta)^{(n-K) / n} \sim 1 / \rho(A)-\delta .
$$

Since $\lim \sup a_{n}^{1 / n}=1 / \rho(A)$, we are done.

Example 1 Graphs on surfaces. Let $\mathcal{G}_{n}^{S}$ be the set of labeled graphs on the vertex set $\{1, \ldots, n\}$ that can be embedded on the surface $S$. McDiarmid [2] showed that $\lim \left|\mathcal{G}_{n}^{S}\right|^{1 / n}$ exists and is independent of $S$. Using this, he obtained some properties of such graphs in both the labeled and unlabeled cases. McDiarmid showed that smoothness allowed one to deduce additional properties and posed the problem of proving smoothness. In a later section we will prove 
Theorem $2 \mathcal{G}_{n}^{S}$ has smooth growth and this remains true if we restrict ourselves to connected graphs. This also remains true if we restrict ourselves to minimal embeddings; that is, graphs not embeddable on a simpler surface.

We have not been able to prove smoothness for unlabelled graphs.

Example 2 Flags on poles. Let $P$ be a set of positive integers. We are given a set $\mathcal{F}$ of flags, each of which has some number of spots. Let $f_{n}$ be the number with $n$ spots. We want to place flags on poles and count the results by total number of spots. The number of flags on a pole must lie in $P$, each pole must have a different number of flags, and there is no order to the poles. Each flag may be used any number of times. If $P(x)=\prod_{n \in P}\left(1+x^{n}\right)=\sum p_{n} x^{n}$ and $F(x)=\sum_{n} f_{n} x^{n}$, then the generating function is $P(F(x))$. Suppose that

- $\operatorname{gcd}\left\{i-j \mid f_{i} f_{j} \neq 0\right\}=1$;

- $\rho(F) \geq 1$;

- for some $d$ and all sufficiently large $n,[n, n+d]$ contains a nonzero $p_{i}$.

Note that $\rho(P)=1$ and $F(\rho(F)) \geq 2$ since $F$ contains at least two nonzero terms. Although $P(x)$ may behave badly on the unit circle, Theorem 1 applies.

Example 3 Functional digraphs. A functional digraph is a digraph in which every vertex has out-degree 1. A functional digraph with vertex set $V$ can be thought of as a function $f: V \rightarrow V$. Some elements of $V$ lie on directed cycles, which include loops and 2 -cycles. When the cyclic edges are deleted, each cyclic element $v$ becomes the root of a tree whose other vertices are noncyclic and whose edges are directed toward $v$. If $C(x)$ is the exponential generating function for directed cycles and $T(x)$ is the exponential generating function for rooted trees, then $C(T(X))$ is the exponential generating function for functional digraphs.

If all functional digraphs are allowed,

$$
C(x)=x+\frac{x^{2}}{2}+\sum_{k=3}^{\infty} \frac{2(n-1) ! x^{n}}{n !}=-2 \ln (1-x)-x-\frac{x^{2}}{2} .
$$

and $T(x)=x e^{T(x)}$. Since $\rho(C)=1, \rho(T)=1 / e$ and $T(1 / e)=1$, Theorem $1(\mathrm{c})$ fails to hold. This leads to the open problem of how to extend the theorem to allow $s=\rho(G)$ in condition (c). The example $F(x)=\frac{1}{1-x}$ and $G(x)=\sum x^{(n !) !} / 2^{n}$, provided by Jason Bell [1], shows that stronger conditions are needed since $a_{(n !) !-1} / a_{(n !) !} \rightarrow 0$.

If we restrict the allowable tree structures, it is quite possible that Theorem 1(c) can be satisfied and so smooth growth follows. Here are some examples.

- If only finitely many tree structures are allowed, $T(x)$ is a polynomial and $r(T)=\infty$. 
- If the trees must be paths, then $t_{n}=n$ ! and so $T(x)=\frac{x}{1-x}$. We have $s=1 / 2$.

- If the trees must be full binary trees, then $T(x)=x+x T(x)^{2} / 2$ and so

$$
T(x)=\frac{1-\sqrt{1-2 x^{2}}}{x} .
$$

Since $\rho(T)=2^{-1 / 2}, T(2 / 3)=1$ and $2 / 3<2^{-1 / 2}$, it follows that (c) is satisfied with $s=2 / 3$.

We may also restrict the allowable cycle lengths. All that is required is that the set $S$ of allowable lengths satisfy Theorem $1(\mathrm{~d})$. Since there will be $2(n-1)$ ! cycles of length $n>2$, it follows that $\rho(C)=1$ still holds.

\section{Proof of Theorem 1}

Notation: For convenience, we let $g(x)=\ln G(x)$ and $\kappa=k / n$. In what follows, there are three types of positive functions depending on $k$ and $n$ :

- $\epsilon \rightarrow 0$ and which we control;

- $\delta \rightarrow 0$ which are not under our control and may depend on $\epsilon$;

- $\nu$ which has subexponential growth, i.e. $\ln \nu / n \rightarrow 0$.

These functions may have different values in different paragraphs. Within a paragraph, they are subscripted if more than one value is needed.

Note that

$$
\frac{a_{n+1}}{a_{n}}=\frac{\sum_{k \leq n+1} f_{k}\left[x^{n+1}\right]\left(G(x)^{k}\right)}{\sum_{k \leq n} f_{k}\left[x^{n}\right]\left(G(x)^{k}\right)},
$$

where the range of $k$ is limited as indicated because $G(x)$ has no constant term.

Step 1. We begin with some simple observations concerning $G$. Since the coefficients of $G(x)$ are nonnegative, $G(r)$ is a strictly increasing function on $(0, \rho(G))$ and so the $s$ mentioned in Theorem 1(c) is well defined. By Theorem 1(b), $G(x)$ has a nonlinear term and so

$$
r g^{\prime}(r)=\frac{\sum_{n>0} n g_{n} r^{n}}{\sum_{n>0} g_{n} r^{n}}
$$

is strictly increasing on $(0, \rho(G))$. Define

$$
L=\lim _{r \rightarrow \rho(G)^{-}} r g^{\prime}(r)
$$

The function $r g^{\prime}(r)$ is a strictly increasing continuous function from $(0, \rho(G))$ onto $(1, L)$ and so $1 / \kappa=r g^{\prime}(r)$ defines $r \in(0, \rho(G))$ as a strictly decreasing continuous function of $\kappa \in(1 / L, 1)$. 
Step 2. We now estimate $\left[x^{n}\right] G(x)^{k}$ and $\left[x^{n+1}\right] G(x)^{k} /\left[x^{n}\right] G(x)^{k}$ by stationary phase when $\kappa$ is on a closed subinterval of $(1 / L, 1)$ and $n \rightarrow \infty$. Theorem $1(\mathrm{~b})$ insures that $|G(z)| \leq G(|z|)$ with equality if and only if $z=|z|$. Thus $|\arg z| \geq \epsilon$ implies that $|G(z)| / G(|z|)<1-\delta$. With $z=r e^{i \theta}$, we have

$$
\left[x^{n}\right] G(x)^{k}=\frac{1}{2 \pi i} \oint \frac{G(z)^{k}}{z^{n+1}} d z=\frac{1}{2 \pi} \int_{-\pi}^{\pi} \exp \left(k g\left(r e^{i \theta}\right)-n i \theta-n \ln r\right) d \theta .
$$

For stationary phase,

$$
0=\frac{d}{d \theta}\left(k g\left(r e^{i \theta}\right)-n i \theta-n \ln r\right)=k i z g^{\prime}(z)-n i
$$

and so

$$
r g^{\prime}(r)=1 / \kappa
$$

Split the right-hand integral in (2.3) into integrals over $|\theta|<\epsilon$ and $\epsilon \leq|\theta| \leq \pi$. Expanding $g\left(r e^{i \theta}\right)$ as a power series in $\theta$ on the first interval, we have

$$
\begin{aligned}
k g\left(r e^{i \theta}\right)-n i \theta-n \ln r & =k g(r)+k i r g^{\prime}(r) \theta+O\left(k \epsilon^{2}\right)-n i \theta-n \ln r \\
& =k g(r)+O\left(k \epsilon^{2}\right)-n \ln r
\end{aligned}
$$

and so

$$
\frac{1}{2 \pi} \int_{-\epsilon}^{\epsilon} \exp \left(k g\left(r e^{i \theta}\right)-n i \theta-n \ln r\right) d \theta=\epsilon \nu_{1} G(r)^{k} / r^{n} .
$$

The integrand in the second integral is bounded in magnitude by $(1-\delta)^{k} G(r)^{k} / r^{n}$. It follows that, if $\epsilon \rightarrow 0$ sufficiently slowly

$$
\left[x^{n}\right] G(x)^{k}=\nu_{2} G(r)^{k} / r^{n} \quad \text { where } \quad 1 / \kappa=r g^{\prime}(r)=r G^{\prime}(r) / G(r)
$$

determines $r>0$.

With the same value of $r$ and $\epsilon$ as in the previous paragraph, we have

$$
\left[x^{n+1}\right] G(x)^{k}=\frac{1}{2 \pi} \int_{-\pi}^{\pi} \frac{1}{r e^{i \theta}} \exp \left(k g\left(r e^{i \theta}\right)-n i \theta-n \ln r\right) d \theta .
$$

On the first interval, namely $|\theta|<\epsilon,\left|e^{i \theta}-1\right| \leq|\theta|<\epsilon$ and so that integral is asymptotic to (2.5) divided by $r$. We bound the integral over the second interval as before. Thus

$$
\frac{\left[x^{n+1}\right] G(x)^{k}}{\left[x^{n}\right] G(x)^{k}} \sim \frac{1}{r} .
$$

Note that since $r$ is a continuous function of $\kappa$,

$$
\frac{\left[x^{n+1}\right] G(x)^{k^{*}}}{\left[x^{n}\right] G(x)^{k^{*}}} \sim \frac{1}{r} \text { provided } k^{*} / n \sim \kappa .
$$


Step 3. We now study the maximum of $f_{k}\left[x^{n}\right] G(x)^{k}$. For the sequence $S$ in Theorem 1(d) let $\delta$ be such that $f_{k} \geq(\rho(F)+\delta)^{-k}$ and set $R=\rho(F)+\delta$. For $\delta$ sufficiently small, it follows from Theorem 1 (c) that $G(t)=R$ has a solution $t<\rho(G)$. It follows that, for $k \in S$,

$$
f_{k}\left[x^{n}\right] G(x)^{k} \geq \nu R^{-k} G(r)^{k} / r^{n}=\nu\left(\frac{(G(r) / R)^{\kappa}}{r}\right)^{n},
$$

where $r$ is given in (2.6) and the function $\nu$ may depend on $\delta$.

We now seek the value $\kappa_{1}$ of $\kappa$ that maximizes $(G(r) / R)^{\kappa} / r$, remembering that $r$ depends on $\kappa$. Differentiating the logarithm of the expression with respect to $\kappa$ and setting it to zero, we obtain

$$
0=\frac{d(\kappa g(r)-\kappa \ln R-\ln r)}{d \kappa}=g(r)-\ln R+\left(\kappa g^{\prime}(r)-1 / r\right) \frac{d r}{d \kappa}=g(r)-\ln R,
$$

where the last equality follows from (2.4). Thus

$$
G(r)=R \quad \text { and, by }(2.4), \quad 1 / \kappa_{1}=r G^{\prime}(r) / G(r)
$$

determine $r$ and a value of $\kappa_{1} n$ near which $f_{k}\left[x^{n}\right] G(x)^{k}$ is a maximum. As $n \rightarrow \infty$, $R \rightarrow \rho(F)$ and $r \rightarrow s$, the value given in Theorem 1(c). For reasons that become apparent later, we write $s=r(1)$. By continuity, when $\left|\kappa-\kappa_{1}\right|<\epsilon$ the values of $r$ and $G(r)$ given by (2.6) are asymptotic to $r(1)$ and $G(r(1))$. Thus we have

$$
f_{k}\left[x^{n}\right] G(x)^{k} \geq\left(\frac{1-\delta}{r(1)}\right)^{n} \quad \text { when } \quad\left|\kappa-\kappa_{1}\right|<\epsilon \text { and } k \in S .
$$

Step 4. We now bound $\sum f_{t}\left[x^{n}\right] G(x)^{t}$ where the sum is over values of $t$ much smaller and much larger than $\kappa n$. There are $C$ and $\delta$ such that $f_{t} \leq C\left(\frac{1+\delta}{\rho(F)}\right)^{t}$. If $u \geq 1$, then

$$
\begin{aligned}
\sum_{t \geq k} f_{t}\left[x^{n}\right] G(x)^{t} & \leq u^{-k} \sum_{t \leq n} f_{t}\left[x^{n}\right] G(x)^{t} u^{t} \\
& \leq C u^{-k} \sum_{t \leq n}\left(\frac{1+\delta}{\rho(F)}\right)^{t}\left[x^{n}\right] G(x)^{t} u^{t} \\
& \leq C(1+\delta)^{n} u^{-k}\left[x^{n}\right]\left(\sum_{t \leq n}(G(x) u / \rho(F))^{t}\right) \\
& =\left[x^{n}\right]\left(\frac{C(1+\delta)^{n} u^{-k}}{1-G(x) u / \rho(F)}\right)
\end{aligned}
$$

where the sums are limited to $t \leq n$ since $G(x)$ has no constant term. Thus

$$
\sum_{t \geq k} f_{t}\left[x^{n}\right] G(x)^{t} \leq \frac{(1+\delta)^{n} \nu}{u^{k} r(u)^{n}}=\frac{(1+\delta)^{n} \nu}{\left(u^{\kappa} r(u)\right)^{n}} \quad \text { where } \quad G(r(u))=\rho(F) / u,
$$


provided $u \geq 1$. Note that $r(1)=s$ and so equals the $r(1)$ introduced in Step 3 . The same result holds for the sum over $t \leq k$ provided $0<u \leq 1$ and such an $r(u)<\rho(G)$ exists. Theorem 1(c) guarantees the existence of $r(u)$ as long as $u$ is not too small.

Step 5. We now show that $u^{\kappa} r(u)$ has a unique global minimum at $u=1$ by computing derivatives with respect to $u$. We have

$$
\frac{d \ln \left(u^{\kappa} r(u)\right)}{d u}=\frac{d \kappa}{d u} \ln u+\frac{\kappa}{u}+\frac{r^{\prime}(u)}{r(u)} .
$$

From the right side of $(2.11), r^{\prime}(u)=-G(r(u)) / u G^{\prime}(r(u))$. From the right side of (2.6), $\kappa=G\left(r(u) / r(u) G^{\prime}(r(u))\right.$. Thus

$$
\frac{d \ln \left(u^{\kappa} r(u)\right)}{d u}=\frac{d \kappa}{d u} \ln u .
$$

By Step 1, $\kappa$ is a decreasing function of $r$. By the right side of (2.11), $r$ is a decreasing function of $u$. Thus $d \kappa / d u>0$. It follows from (2.12) that $u^{\kappa} r(u)$ has a unique global minimum at $u=1$.

Step 6. We now put it all together. Recall $\kappa_{1}$ from Step 3. Let $\epsilon_{1}$ be such that there is a $k \in S$ such that $\left|\kappa_{1}-k / n\right|<\epsilon_{1}$. Let $\delta_{1}$ be such that $f_{k}\left[x^{n}\right] G(x)^{k} \geq\left(\frac{1-\delta_{1}}{r(1)}\right)^{n}$ at this value of $k$. Choose $\epsilon_{2}>\delta_{1}$. From (2.11) and (2.12), there is a $\delta_{2}>\epsilon_{1}$ such that

$$
\sum_{\left|\kappa_{1}-t / n\right| \geq \delta_{2}} f_{t}\left[x^{n}\right] G(x)^{t} \leq\left(\frac{1-\epsilon_{2}}{r(1)}\right)^{n} .
$$

Thus

$$
\frac{\sum_{k \leq n+1} f_{k}\left[x^{n+1}\right]\left(G(x)^{k}\right)}{\sum_{k \leq n} f_{k}\left[x^{n}\right]\left(G(x)^{k}\right)} \sim \frac{\sum_{\left|\kappa_{1}-k / n\right|<\delta_{2}} f_{k}\left[x^{n+1}\right]\left(G(x)^{k}\right)}{\sum_{\left|\kappa_{1}-k / n\right|<\delta_{2}} f_{k}\left[x^{n}\right]\left(G(x)^{k}\right)} .
$$

Since the ratio of terms with the same $k$ in the interval $\left|\kappa_{1}-k / n\right|<\delta_{2}$ are asymptotic to $1 / r(1)$ by (2.8), the proof is complete.

\section{Proof of Theorem 2}

All generating functions in this section are exponential.

Let $\mathcal{F}_{n}^{S}$ be the set of graphs embeddable on the surface $S$ and for which all vertices have degree at least two. There are two cases, depending on whether or not we limit our attention to connected graphs. Let $F^{S}(x)=\sum\left|\mathcal{F}_{n}^{S}\right| x^{n} / n !=\sum f_{n} x^{n}$. We will show that $\lim _{n \rightarrow \infty} f_{n}^{1 / n}$ exists, lies in $(0,1)$ and is independent of $S$. When $S$ is the plane, Theorems 3.3 and 2.2 in [3] dispose of the general and connected cases, respectively. For other surfaces, our proof is essentially a copy of McDiarmid's proof of his Theorem 2.1 in [2]. Consequently we assume the reader has a copy of that proof available. Our $\mathcal{F}_{n}^{S}$ plays the same role as his $\mathcal{A}_{n}^{S}$. The key observation is that his Lemma 4.2 remains valid. 
His proof involves cutting a graph along a cycle. Suppose $\{x, y\}$ is an edge in the cycle. As a result of the cut, he replaces $x$ with two vertices, say $x^{\prime}$ and $x^{\prime \prime}$ and likewise for $y$. When cutting, we add the edges $\left\{x^{\prime}, y^{\prime}\right\}$ and $\left\{x^{\prime \prime}, y^{\prime \prime}\right\}$, thereby guaranteeing that all vertices have degree at least two after cutting. The remainder of McDiarmid's proof of Theorem 2.1 is essentially unchanged. For the connected case, it suffices to observe that McDiarmid's $\mathcal{B}$ contains at most two components.

We begin with the connected case of Theorem 2. Set $f_{0}=f_{1}=0$. Let $R(x)$ and $T(x)$ be the generating functions for rooted and unrooted labelled trees, respectively. Recall that $\rho(R)=1 / e$ and $R(1 / e)=1$, results deducible from the functional equation $R(x)=x e^{R(x)}$. Also, $\rho(T)=\rho(R)$ since $R(x)=x T^{\prime}(x)$. The generating function for connected graphs embeddable on $S$ is $F^{S}(R(x))+T(x)$. To apply Theorem 1 to $F^{S}(R(x))$, we must verify condition (c). Since $R(\rho(R))=1$ and $\rho\left(F^{S}\right)<1$, the condition follows.

We claim $\gamma=\rho\left(F^{S}(R)\right)<1 / e=\rho(T)$. If so smoothness then follows from the smoothness of $F^{S}(R(x))$ since $\lim \left(\left[x^{n}\right] F^{S}(R(x))\right)^{1 / n}=1 / \gamma$ and $\lim \sup \left(\left[x^{n}\right] T(x)\right)^{1 / n}=$ $e<1 / \gamma$. To prove the claim, note that $\gamma$ is the solution to $R(\gamma)=\rho\left(F^{S}\right)$, that $\rho\left(F^{S}\right)<1$ and that $R(\rho(T))=1$.

We now prove the unrestricted case. Set $f_{0}=1$ and $f_{1}=0$. If $U(x)$ is the exponential generating function for unrooted labelled forests, then $F^{S}(T(x)) U(x)$ counts all graphs

embeddable on $S$. Since unrooted forests are counted by $e^{T(x)}$, we have $\rho(U)=\rho(T)=$ 1/e. Theorem 1 can be applied to $F^{S}(T(x))$ to obtain smooth growth of those coefficients. We now apply a problem of Pólya and Szegö [4] to the product of $B(x)=F^{S}(T(x))$ and $U(x)$. It concludes that $\left[x^{n}\right](B(x) U(x)) \sim\left(\left[x^{n}\right] B(x)\right) U(\rho(B))$. The only condition that is not obvious is $\rho(B)<\rho(U)$, but this was proved in the previous paragraph since $\rho(U)=1 / e$.

The minimal embedding result follows from Theorem 2.5 of [2].

Acknowledgement. We thank Colin McDiarmid for his smoothness conjecture which led to this paper and for helpful comments on our proof of Theorem 2.

\section{References}

[1] J. Bell, private communication.

[2] C. McDiarmid, Random graphs on surfaces, J. Combin. Theory Ser. B, to appear. Available at www.crm.es/Publications/06/Pr722.pdf

[3] C. McDiarmid, A. Steger and D.J.A. Welsh, Random planar graphs, J. Combin. Theory Ser. B, 93 (2005) 187-205.

[4] G. Pólya and G. Szegö, Problems and Theorems in Analysis, Springer (1998), vol. I, part I, \#178. A translation of Aufgaben und Lehrsätze aus der Analysis. 